\title{
Uso problemático de internet en estudiantes universitarios: factores asociados y diferencias de género
}

\author{
Problematic Internet Use in University Students: \\ associated factors and differences of gender
}

\author{
Tania Fernández-Villa*, Juan Alguacil OJeda**,***, Ana Almaraz Gómez****, José María \\ Cancela Carral *****, Miguel Delgado-Rodríguez*********, Miguel García-Martín*******, \\ Eladio JimÉnez-Mejías $* * * * * * *$, Javier LlorcA***********, Antonio José Molina*,*********, Rocío \\ Ortíz MoncadA $* * * * * * * * * *$, Luiz Félix Valero-Juan***********, Vicente Martín*,**,*********
}

*Departamento de Ciencias Biomédicas. Área de Medicina Preventiva y Salud Pública. Universidad de León; **Centro de Investigación Biomédica en Red de Epidemiología y Salud Pública (CIBERESP); ***Centro de Investigación en Salud y Medio Ambiente (CYSMA). Universidad de Huelva; ****Departamento de Medicina Preventiva y Salud Pública, Universidad de Valladolid; *****Grupo de Investigación Healthy Fit. Facultad de Ciencias de la Educación y el Deporte. Universidad de Vigo; ******Área de Medicina Preventiva y Salud Pública. Universidad de Jaén; *******Departamento de Medicina Preventiva y Salud Pública. Universidad de Granada; ********Universidad de Cantabria - IDIVAL; *********Grupo de Investigación de Interacción Gen-Ambiente-Salud (GIIGAS). Universidad de León; **********Grupo de Investigación Alimentación y Nutrición, Área de Medicina Preventiva y Salud Pública. Universidad de Alicante.; ***********Área de Medicina Preventiva y Salud Pública. Universidad de Salamanca.

\section{Resumen}

El objetivo del presente trabajo es realizar un análisis descriptivo del uso problemático de Internet en estudiantes universitarios, evaluando la posible asociación con problemas de salud y conductas adictivas, así como diferencias de género en los tipos utilización. Un total de 2780 alumnos participaron en el estudio entre los años 2011 - 2014, siendo un $29 \%$ varones (edad $20.8 \pm 5.1$ años) y un $71 \%$ mujeres (edad de $20.3 \pm 4.4$ años). La prevalencia de uso problemático de Internet evaluada mediante el Internet Addiction Test fue del 6.08\%. Ser menor de 21 años y cursar titulaciones diferentes a ciencias de la salud fueron factores asociados a una mayor frecuencia de este problema, no existiendo diferencias en función del sexo o tipo de domicilio. Los resultados muestran una asociación significativa con algunos problemas de salud (migrañas, dolor lumbar, sobrepeso u obesidad, descanso insuficiente), aspectos psicológicos (riesgo de trastornos de la conducta alimentaria, riesgo de trastorno mental, depresión), problemas familiares y discriminación; no encontrándose asociaciones con consumo de sustancias adictivas (alcohol, tabaco o cannabis). Respecto al tiempo de uso de Internet, las horas de conexión semanales fueron significativamente mayores en las mujeres que en los hombres, tanto en el tiempo total como por motivos de ocio. El análisis del perfil de utilización en usuarios problemáticos reveló que los varones se relacionan más con aspectos de ocio como los juegos o las compras online y las mujeres con aspectos de socialización, como el chat o las redes sociales.

Palabras clave: Uso problemático de Internet, Epidemiología, prevalencia, Estudiantes universitarios.

\begin{abstract}
The aim of this paper is to make a descriptive analysis of Problematic Internet Use in college students, evaluating the possible association with health problems and addictive behaviors, as well as gender differences in user types. A total of 2,780 students participated in the study between 2011 and 2014, 29\% of them being males (age $20.8 \pm$ 5.1 years) and $71 \%$ females (age $20.3 \pm 4.4$ years). The prevalence of Problematic Internet Use (PIU) assessed by the Internet Addiction Test was $6.08 \%$. Being under 21 years of age and studying for degrees in subjects other than the health sciences were associated factors with a higher frequency of this problem, no differences by gender or type of address were found. The results show a significant association with some health problems (migraines, back pain, excess weight or obesity, insufficient rest), psychological aspects (risk of eating disorders, risk of mental disorder, depression), family problems and discrimination; with no associations with substance use (alcohol, cannabis or tobacco) being found. Concerning the time of Internet use, weekly hours were significantly higher in women than in men, both the total time as for leisure. The analysis of the profile use in problematic users revealed that males are related to aspects of entertainment such as games or shopping online and females are related to aspects of socialization, such as chats and social networks.
\end{abstract}

Keywords: Problematic Internet Use, Epidemiology, Prevalence, University Students

Recibido: Julio 2014; Aceptado: Abril 2015

Enviar correspondencia a:

Tania Fernández Villa, Universidad de León, Facultad Universitaria de Ciencias de la Salud, Campus de Vegazana s/n, 24071, León, España. E-mail: tferv@unileon.es. 


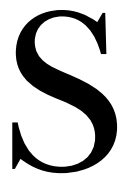
egún los últimos datos del Internet World Stats, más de 2405 millones de personas en el mundo y 518 millones de personas en Europa son usuarias de Internet. España se sitúa entre los 20 países de mayor utilización a nivel mundial, ocupando el séptimo puesto en la Unión Europea con más de 31 millones de usuarios (Internet World Stats, 2014).

Esta herramienta que nació a finales de los 60 en Estados Unidos, ante la necesidad de una red de comunicación entre agencias del gobierno como sistema defensivo, comenzó su diversificación en los años 80 a otras áreas académicas y científicas y en los últimos 20 años hemos asistido a su crecimiento exponencial (Glowniag, 1998). En nuestro país, este crecimiento se ha observado especialmente en la última década, pasando de un 27\% de usuarios de Internet en 1993, al $64.5 \%$ en 2013, según los datos reportados por la Encuesta General de Medios (AIMC, 2013).

Este crecimiento explosivo guarda relación con las numerosas ventajas que aporta la red, como la inmediatez en la información y comunicación, la socialización y la utilización como medio de ocio y entretenimiento, especialmente entre los más jóvenes; permitiendo además mantener el anonimato, la socialización virtual o la construcción de identidades, facilitando la comunicación de personas tímidas o introvertidas (Muñoz-Rivas, Navarro y Ortega, 2003).

Pero este medio, que se ha convertido en un elemento cotidiano en nuestras vidas no está exento de problemas. $\mathrm{Su}$ utilización requiere tiempo y la falta de autocontrol puede ocasionar evasión de la vida real e incluso estados de dependencia o adicción (Didia, Dorphinghaus, Maggi y Haro, 2009; Echeburúa y Corral, 2010; Gracia, Vigo, Fernández y Marco, 2002; Young, 1998).

En muchas ocasiones, el atractivo de la red podría incluso compararse con las propiedades reforzadoras de las sustancias adictivas, motivo por el cual, algunos autores han descrito síntomas o perfiles psicológicos derivados del uso excesivo de la red, similares a los producidos en personas adictas a sustancias (como estrés, ansiedad o inquietud ante la falta de conexión) (Lam-Figueroa et al., 2011; Young, 1998).

Términos como "Adicción a Internet", "Uso Patológico de Internet", "Uso Compulsivo de Internet", "Netadicción" o "Ciberadicción", han sido ampliamente utilizados en la literatura científica para referirse a este problema (Douglas et al., 2008; Luengo, 2004; Young, 1998; Young, 2010;). Sin embargo, la adicción a Internet, que se enmarca dentro de las denominadas adicciones comportamentales o psicológicas, no se encuentra registrada como tal en ningún manual diagnóstico (American Psichiatric Association, 2003; Organización Mundial de la Salud, 1992), habiendo sido considerado únicamente el juego online patológico como trastorno relacionado con el uso de Internet en la reciente publicación del DSM-V (American Psychiatric Association, 2014; Carbonell, 2014).
En un estudio descriptivo llevado a cabo en población adolescente de once países europeos, se encontró una asociación directa con el Uso Problemático de Internet (UPI), en aquellos que presentaban síntomas depresivos, ansiedad y alteraciones para mantener relaciones personales en la vida real, especialmente los varones (Kaess et al.,2014). Además de esto, algunos autores han encontrado una asociación con otros problemas de salud, tales como deprivación del sueño, trastornos de la conducta alimentaria, migrañas o dolores de espalda (Didia et al., 2009; Pezoa-Jares, Espinoza-Luna y Vasquez-Medina, 2012; Spada, 2014)

Por otra parte, entorno al $12 \%$ de los adultos adictos a internet presentan también problemas de dependencia o adicción al alcohol y un 5\% a las drogas en general, según muestra la revisión realizada por Sussman, Lisha y Griffiths (2011). Este aspecto ha sido evidenciado en el reciente estudio de Secades-Villa et al. (2014) llevado a cabo en población adolescente europea, en el que encontró una relación estadísticamente significativa entre las horas de conexión a internet y la frecuencia de consumo de alcohol, tabaco, cannabis y otras drogas ilegales.

El perfil de mayor riesgo de UPI, se enmarca en una persona de sexo masculino, con edad inferior a 21 años, baja autoestima y con domicilio lejos del ambiente familiar, siendo por tanto más vulnerable hacia los problemas anteriormente descritos. (Sánchez-Carbonell, Beranuy, Castellana, Chamarro y Oberst, 2008; Frangos, Frangos y Kiohos, 2010; Muñoz-Rivas et al., 2003).

Respecto a los tipos de utilización, diferencias de género han sido descritas en la literatura científica, siendo los varones los que más horas invierten a la semana, especialmente en relación al uso del correo electrónico, juegos online y visita de páginas web (Muñoz-Rivas et al., 2003) y siendo las mujeres las que más utilización hacen de chats o redes sociales (Andreassen, Torsheim, Brunborg y Pallesen, 2012).

El análisis de los comportamientos relacionados con el uso de Internet tiene un gran interés especialmente en población joven, puesto que los adolescentes y estudiantes universitarios son los grupos más susceptibles de sufrir pérdida del autocontrol (Hicks y Heastie, 2008; Kandell, 1998; Ledo-Varela et al., 2011).

La etapa universitaria es un periodo de transición que en muchos casos implica la independencia del núcleo familiar, estrés ante la nueva situación o búsqueda de nuevas amistades, motivos que pueden interferir en el uso de la red, así como en cambios en estilos de vida que pueden repercutir en la salud futura. Las prevalencias de UPI en esta población rondan entre el $6-40 \%$, existiendo controversia debido a las diferencias metodológicas de los estudios publicados (Pezoa-Jares et al., 2012; Sussman et al., 2011).

Por ello, la Universidad de León decidió poner en marcha en el año 2011 el proyecto uniHcos, con el fin de analizar la influencia de la etapa universitaria en los diferentes estilos de vida, contando con la colaboración actualmente 
de otras ocho universidades españolas (Fernández-Villa et al., 2013).

El presente trabajo, que se enmarca dentro de dicho proyecto, es un estudio epidemiológico descriptivo que tiene como novedad el amplio análisis propuesto de posibles factores o comportamientos que pueden estar asociados al UPI, planteándose tres hipótesis principales: (a) que existe una mayor asociación en personas jóvenes, de menos de 21 años, que viven fuera del hogar familiar y que presentan baja autoestima o estados de depresión o ansiedad, siendo el sexo masculino el grupo más vulnerable; (b) que existe una asociación también no solo con problemas de salud tanto físicos como psicológicos, sino con el uso de sustancias adictivas tales como alcohol, tabaco y otras drogas ilegales y; (c) que existen diferencias de género en los perfiles de utilización de Internet.

Con el fin de contrastar estas hipótesis, se establecen objetivos principales: (a) evaluar la relación entre el UPI y aspectos sociodemográficos; (b) analizar la posible asociación con problemas de salud tanto físicos como psicológicos, así como con consumo de sustancias adictivas (alcohol, tabaco y cannabis) y (c) describir los perfiles de utilización de Internet en busca de diferencias de género.

\section{Método}

\section{Diseño}

Estudio epidemiológico observacional, descriptivo, transversal.

\section{Participantes}

La población diana fueron todos los universitarios de primer año y primera matrícula de Grado, de cualquier titulación impartida en las universidades colaboradoras en el proyecto uniHcos (Fernández-Villa et al., 2013), desde octubre del 2011 hasta abril del 2014. Los estudiantes fueron reclutados a partir de una invitación enviada a los correos electrónicos institucionales, participando de forma totalmente voluntaria y sin recibir ninguna compensación por la encuesta realizada un total de 2780 alumnos $(3.5 \%)$. Un $28.8 \%$ eran varones (edad media $20.8 \pm 5.1$ años) y un $71.2 \%$ mujeres (edad media $20.3 \pm 4.4$ años), existiendo representación de todas las universidades participantes (2\% de Alicante, $2 \%$ de Cantabria, 37\% de Granada, $4 \%$ de Huelva, $8 \%$ de Jaén, $17 \%$ de León, $14 \%$ de Salamanca, 3\% de Valladolid y $14 \%$ de Vigo). No fue necesario prescindir de ninguna encuesta en el análisis, ya que los ítems del cuestionario eran de respuesta obligatoria y por lo tanto no hubo errores o datos ausentes.

\section{Instrumentos}

Mediante un cuestionario online auto-administrado de 373 ítems se recogió información sobre: aspectos sociodemográficos (edad, sexo, titulación, tipo de domicilio, uni- versidad) consumo de sustancias (alcohol, tabaco y cannabis), características físicas y psicológicas, así como aspectos relacionados con el uso de Internet (horas diarias de conexión a la semana, y tipo de utilización). Los instrumentos utilizados para ello fueron:

Test APGAR (Adaptability, Partnertship, Growth, Affection, Resolve). Es un breve cuestionario unidimensional formado por cinco preguntas con escala Likert (0 - 2 puntos) que sirve para evaluar la funcionalidad o disfunción familiar (alfa de Cronbach de .84). Una puntuación superior a seis puntos indica una buena funcionalidad familiar, mientras que una puntuación menor o igual a seis puntos indica una disfunción moderada - grave (Bellón, Delgado, Del Castillo y Lardelli, 1996; Smilkstein, Ashworth y Montano, 1982).

Cuestionario SCOFF (Sick, Control, Out weight, Fat and Food). Consta de cinco preguntas dicotómicas, de modo que cada respuesta positiva equivale a 1 punto. Un total de dos o más puntos determina mayor probabilidad de conducta alimentaria de riesgo, con una sensibilidad de .98 y una especificidad .94. (Garcia-Campayo et al., 2005; Morgan, Reid y Lacey, 1999).

Test GHQ-12 (General Health Questionnaire). Compuesto por 12 ítems, seis de ellos de orientación positiva y los otros seis de orientación negativa. Presenta una buena fiabilidad en los diferentes estudios realizados con alfas de Cronbach que varían entre .82 y .86 (Goldberg et al., 1997). Se evalúa según una escala Likert con puntuación dicotómica (0-0-1-1). Un resultado final con tres o más puntos corresponde a un mayor riesgo de problemas psicológicos (Rocha, Pérez, Rodríguez, Borrell y Obiols, 2011)

Test AUDIT (Alcohol Use Disorders Identification Test). Cuestionario formado por 10 ítems que son evaluados mediante una escala Likert de cinco posibilidades ( 0 - 4 puntos) a excepción de los ítems 9 y 10 cuya escala es de tres opciones $(0-2-4$ puntos $)$. Un valor de ocho o más puntos indica un consumo problemático de alcohol, con una sensibilidad de .90 y una especificidad de .80. (Saunders, Aasland, Babor, de la Fuente y Grant, 1993).

Cuestionario IAT (Internet Addiction Test). Fue elaborado en 1998 por Kimberly Young para analizar el grado en que el uso de Internet afecta a la vida cotidiana, la vida social, a la productividad, al sueño y los sentimientos de la persona (Young, 1998). En las diferentes validaciones publicadas de este cuestionario, han sido encontradas entre una y seis dimensiones diferentes, con un alfa de Cronbach cercano a .90. Consta de 20 ítems evaluados mediante una escala Likert de cinco puntos ( 0 = Nunca; 1 = Casi nunca; 2 $=$ Ocasionalmente; $3=$ Frecuentemente; $4=$ A menudo; $5=$ Siempre). Dada la controversia acerca del número de factores existente, para el presente trabajo hemos decido analizar el UPI de acuerdo al punto de corte de 40, considerando usuarios problemáticos aquellos que obtienen 40 puntos o más (Jelenchick, Becker y Moreno, 2012). 


\section{Procedimiento}

El presente trabajo se enmarca dentro del Proyecto uniHcos, cuyo diseño es multicéntrico de cohorte prospectivo. Todos los estudiantes universitarios que cumplen los criterios de inclusión son invitados a colaborar a través del correo electrónico institucional, realizando posteriormente un seguimiento bienal a todos los participantes. En el estudio descriptivo aquí presentado, se ha tenido cuenta la primera encuesta realizada por los estudiantes de las tres primeras oleadas (desde 2011-2014).

Todos aquellos que desearon colaborar, rellenaron previo a la encuesta un consentimiento informado online. Una vez aceptado, eran dirigidos al cuestionario anteriormente citado, gestionado a partir de la plataforma SphinxOnline ${ }^{\circledR}$, la cual permite mantener el anonimato de los participantes, cumpliendo con la normativa vigente de la Ley Orgánica de Protección de Datos de Carácter Personal (Boletín Oficial del Estado, 1999). Además de ello, se cuenta con la aprobación de los Comités de Ética de las universidades colaboradoras para la utilización de datos personales de los estudiantes.

\section{Análisis Estadístico}

Se utilizó como variable dependiente la categorización dicotómica del uso de Internet, y como variables independientes el resto de información recogida (problemas de salud, aspectos físicos y psicológicos, consumo de sustancias y aspectos relacionados con el uso de Internet). En el análisis descriptivo de variables categóricas se calcularon las prevalencias con sus respectivos intervalos de confianza del 95\%, mientras que en las variables numéricas fueron analizadas mediante el cálculo de medias y desviaciones estándar. La relación entre el uso problemático de Internet y otras variables fue establecida mediante modelos de regresión logística bivariante y multivariante, calculándose Odds Ratio con sus respectivos intervalos de confianza del $95 \%$, realizando los ajustes por Sexo, Edad y Titulación. Se utilizó el paquete estadístico STATA 13 para todos los análisis (Stata Corp, 2013).

\section{Resultados}

\section{Aspectos Generales}

La prevalencia de UPI en la muestra fue del $6.04 \%$, no existiendo diferencias estadísticamente significativas por sexo o tipo de domicilio. Respecto a la edad y a la titulación, la Tabla 1 muestra como los menores de 21 años y aquellos que cursan titulaciones diferentes a ciencias de la salud presentan Odds ratios (OR) próximas a 1.5 tanto en el análisis crudo, como en el ajustado.

\section{Asociación con Problemas de Salud y Aspectos Psi- cosociales}

En la Tabla 2 podemos observar que existe una mayor prevalencia de UPI en personas que presentan dolor de espalda a nivel lumbar y migrañas, no encontrándose asociación significativa con el dolor cervical.

La evaluación de la constitución física mediante el índice de masa corporal, muestra una asociación del UPI con sobrepeso u obesidad, siendo este problema casi 2.5 veces más frecuente en personas con un índice de masa corporal superior a $30 \mathrm{Kg} / \mathrm{m}^{2}(\mathrm{p}=.009)$.

Características Sociodemográficas de los Usuarios Problemáticos.

\begin{tabular}{|c|c|c|c|c|c|c|c|c|c|c|c|}
\hline & \multicolumn{11}{|c|}{ Uso Problemático de Internet } \\
\hline & $\mathbf{N}$ & $\mathbf{n}$ & $\%$ & $\mathrm{ORc}^{1}$ & IC95\% & B & $p$ & $\mathbf{O R a}{ }^{2}$ & IC95\% & B & $p$ \\
\hline \multicolumn{12}{|l|}{ Sexo } \\
\hline Mujer & 1979 & 118 & 6.0 & 1 & & & & 1 & & & \\
\hline Hombre & 801 & 50 & 6.3 & 1.05 & $.75-1.48$ & .049 & .779 & 1.03 & $.74-1.47$ & .039 & .823 \\
\hline \multicolumn{12}{|l|}{ Edad } \\
\hline$\geq 21$ años & 2001 & 37 & 4.5 & 1 & & & & 1 & & & \\
\hline$<21$ años & 816 & 168 & 6.7 & 1.50 & $1.03-2.19$ & .409 & .032 & 1.52 & $1.05-2.22$ & .421 & .028 \\
\hline \multicolumn{12}{|l|}{ Titulación } \\
\hline Ciencias Salud & 726 & 33 & 4.6 & 1 & & & & 1 & & & \\
\hline Otras titulaciones & 2054 & 135 & 6.6 & 1.48 & $.99-2.18$ & .390 & .050 & 1.49 & $1.01-2.21$ & .399 & .046 \\
\hline \multicolumn{12}{|l|}{ Tipo de Domicilio } \\
\hline Domicilio Familiar & 1190 & 79 & 6.6 & 1 & & & & 1 & & & \\
\hline Colegio Mayor / Residencia & 300 & 16 & 5.3 & .79 & $.45-1.37$ & -.236 & .402 & .72 & $.41-1.25$ & -.330 & .246 \\
\hline Otros & 1384 & 168 & 6.0 & .84 & $.61-1.17$ & -.169 & .312 & .87 & $.62-1.21$ & -.131 & .402 \\
\hline
\end{tabular}

Nota. ${ }^{1} \mathrm{ORc}=$ Odds Ratio crudo $;{ }^{2} \mathrm{ORa}=$ Odds Ratio ajustado por Sexo, Edad y Titulación 
Por otra parte, aspectos como un mal estado de salud percibido, riesgo de trastornos de la conducta alimentaria analizado mediante el cuestionario SCOFF o depresión, también se asociaron positivamente con el UPI en ambos análisis, destacando una prevalencia casi cuatro veces superior de UPI en los encuestados con riesgo de problemas de salud mental evaluados mediante el GHQ-12 (ORa = 3.58; $\mathrm{p}=.000)$.

Tabla 2

Relación del Uso Problemático de Internet con problemas de salud y aspectos psicológicos.

\begin{tabular}{|c|c|c|c|c|c|c|c|c|c|c|c|}
\hline & \multicolumn{11}{|c|}{ Uso Problemático de Internet } \\
\hline & $\mathbf{N}$ & $\mathbf{n}$ & $\%$ & ORc ${ }^{1}$ & IC95\% & B & $p$ & ORa ${ }^{2}$ & IC95\% & B & $p$ \\
\hline \multicolumn{12}{|l|}{ Dolor cervical } \\
\hline No & 2493 & 150 & 6.0 & 1 & & & & 1 & & & \\
\hline Sí & 287 & 18 & 6.3 & 1.04 & $.63-1.73$ & .044 & .864 & 1.09 & $.66-1.82$ & .090 & .727 \\
\hline \multicolumn{12}{|l|}{ Dolor lumbar } \\
\hline No & 2427 & 138 & 5.7 & 1 & & & & 1 & & & \\
\hline Sí & 353 & 30 & 8.5 & 1.54 & $1.02-2.33$ & .432 & .040 & 1.60 & $1.06-2.43$ & .472 & .026 \\
\hline \multicolumn{12}{|l|}{ Migrañas } \\
\hline No & 2284 & 129 & 5.7 & 1 & & & & 1 & & & \\
\hline Sí & 496 & 39 & 7.9 & 1.43 & $.98-2.07$ & .355 & .062 & 1.48 & $1.01-2.17$ & .394 & .042 \\
\hline \multicolumn{12}{|l|}{$\mathrm{IMC}^{3}$} \\
\hline Normopeso & 2005 & 112 & 5.6 & 1 & & & & 1 & & & \\
\hline Bajo peso & 267 & 16 & 6.0 & 1.08 & $.63-1.85$ & .746 & .787 & 1.05 & $.61-1.82$ & .055 & .854 \\
\hline Sobrepeso / Obesidad & 508 & 40 & 7.9 & 1.44 & $.99-2.10$ & .368 & .054 & 1.53 & $1.04-2.24$ & .425 & .029 \\
\hline \multicolumn{12}{|l|}{ Salud Percibida } \\
\hline Buena/Muy Buena & 2206 & 115 & 5.2 & 1 & & & & 1 & & & \\
\hline Regular o Peor & 574 & 53 & 9.2 & 1.85 & $1.32-2.60$ & .615 & .000 & 1.90 & $1.35-2.68$ & .643 & .000 \\
\hline \multicolumn{12}{|l|}{ Scoff } \\
\hline No riesgo & 2239 & 116 & 5.2 & 1 & & & & 1 & & & \\
\hline En riesgo & 541 & 52 & 9.6 & 1.95 & $1.38-2.74$ & .666 & .000 & 1.94 & $1.37-2.74$ & .660 & .000 \\
\hline \multicolumn{12}{|l|}{ Ghq_12 } \\
\hline No riesgo & 1266 & 34 & 2.7 & 1 & & & & 1 & & & \\
\hline En Riesgo & 1514 & 134 & 8.9 & 3.52 & $2.40-5.17$ & 1.258 & .000 & 3.58 & $2.44-5.27$ & 1.276 & .000 \\
\hline \multicolumn{12}{|l|}{ Depresión } \\
\hline No & 2432 & 131 & 5.4 & 1 & & & & 1 & & & \\
\hline Sí & 348 & 37 & 10.6 & 2.09 & $1.42-3.07$ & .737 & .000 & 2.22 & $1.50-3.28$ & .799 & .000 \\
\hline \multicolumn{12}{|l|}{ Apgar } \\
\hline Normofuncional & 2067 & 103 & 5.0 & 1 & & & & 1 & & & \\
\hline Disfuncional & 713 & 65 & 9.1 & 1.91 & $1.38-2.64$ & .649 & .000 & 1.87 & $1.35-2.59$ & .627 & .000 \\
\hline \multicolumn{12}{|l|}{ Discriminación } \\
\hline No & 2354 & 115 & 4.9 & 1 & & & & 1 & & & \\
\hline Sí & 426 & 53 & 12.4 & 2.77 & $1.96-3.90$ & 1.018 & .000 & 2.78 & $1.97-3.93$ & 1.023 & .000 \\
\hline \multicolumn{12}{|l|}{ Descanso } \\
\hline Sí & 1584 & 66 & 4.2 & 1 & & & & 1 & & & \\
\hline No & 1196 & 102 & 8.5 & 2.14 & $1.56-2.95$ & .763 & .000 & 2.17 & $1.58-3.00$ & .777 & .000 \\
\hline
\end{tabular}

Nota. ${ }^{1}$ ORc $=$ Odds Ratio crudo ; ${ }^{2}$ ORa= Odds Ratio ajustado por Sexo, Edad y Titulación.; IMC = Índice de Masa Corporal en Kg/m2 considerando Infrapeso $\leq 18,5 \mathrm{Kg} / \mathrm{m} 2$ y Sobrepeso/Obesidad $\geq 25 \mathrm{Kg} / \mathrm{m} 2$, siendo el resto de valores evaluados como Normopeso. 
Nuestros resultados muestran además que las relaciones sociales y familiares son relevantes en los problemas relacionados con el uso de Internet, puesto que los estudiantes que presentan una disfunción familiar, ya sea moderada o grave (APGAR positivo), o algún tipo de discriminación, duplican y hasta triplican la prevalencia de UPI respectivamente.

También es destacable el papel de un buen descanso, puesto que aquellas personas que refieren un descanso inadecuado duplican la frecuencia de los problemas derivados del uso de Internet $(\mathrm{p}=.000)$, existiendo diferencias estadísticamente significativas en cuanto a las horas diarias de sueño (usuarios normales: $7.6 \pm 1.2 \mathrm{~h} /$ día; Usuarios problemáticos: $7.3 \pm 1.5 \mathrm{~h} /$ día ; $\mathrm{p}=.003)$.

\section{Asociación con el Consumo de drogas Legales e Ilegales}

En relación al consumo de sustancias adictivas (alcohol, tabaco y cannabis), no se ha encontrado asociación estadísticamente significativa ni en los análisis crudos ni en los ajustados, tal como muestra la Tabla 3. Sin embargo, si evaluamos el riesgo de problemas de consumo de alcohol mediante el test AUDIT, encontramos dos veces más prevalencia de UPI en los estudiantes con un AUDIT positivo.

\section{Tipos de utilización de Internet y Diferencias de Género}

En el análisis global de los distintos tipos de utilización de Internet, encontramos que el uso del chat o las redes sociales se comporta como un factor de riesgo para el UPI, mientras que el uso del email se relacionó con una menor prevalencia de este problema, no mostrando asociación significativa el resto de usos (Tabla 4).

$\mathrm{Si}$ analizamos el perfil de las personas con criterios de UPI, observamos diferencias de género estadísticamente significativas, tal como muestra la Tabla 5. Las mujeres que utilizan las redes sociales o los chats presentan mayor frecuencia de este problema, mientras que en los varones la mayor prevalencia de UPI se estableció en aquellos que juegan o compran online. La frecuencia de problemas derivados de Internet en relación a las compras online en los hombres ha de ser analizada con cautela dado el pequeño tamaño muestral.

En cuanto al tiempo de utilización, hemos encontrado una media de $32.2 \pm 24.3 \mathrm{~h}$ semanales de conexión en usuarios normales y de $52.7 \pm 31.7$ h en usuarios problemáticos, siendo especialmente relevante el tiempo dedicado a actividades de ocio. Nuestros resultados revelan que son las mujeres las que más horas pasan conectadas a la red (Figura 1), observándose diferencias significativas tanto en el tiempo de conexión global como por ocio entre los usuarios que no mostraron un uso problemático y los problemáticos, no existiendo tal diferencia en relación al tiempo utilizado para actividades relacionadas con el estudio o el trabajo.

Tabla 3

Relación del Uso Problemático de Internet con el consumo de sustancias adictivas en el último mes.

\begin{tabular}{|c|c|c|c|c|c|c|c|c|c|c|c|}
\hline & \multirow[b]{2}{*}{$\mathbf{N}$} & \multicolumn{10}{|c|}{ Uso Problemático de Internet } \\
\hline & & $\mathbf{n}$ & $\%$ & ORc ${ }^{1}$ & $\mathrm{IC95 \%}$ & B & $\mathbf{p}$ & ORa ${ }^{2}$ & $\mathrm{IC} 95 \%$ & B & $\mathbf{p}$ \\
\hline \multicolumn{12}{|l|}{ Cannabis } \\
\hline No & 1638 & 101 & 6.2 & 1 & & & & 1 & & & \\
\hline Sí & 1142 & 67 & 5.9 & .95 & $.69-1.30$ & -.053 & .745 & .98 & $.71-1.35$ & -.020 & .902 \\
\hline \multicolumn{12}{|l|}{ Tabaco } \\
\hline No & 2121 & 126 & 5.9 & 1 & & & & 1 & & & \\
\hline Sí & 659 & 42 & 6.3 & 1.08 & $.75-1.55$ & .785 & .689 & 1.12 & $.78-1.62$ & .117 & .530 \\
\hline \multicolumn{12}{|l|}{ Alcohol } \\
\hline No & 576 & 36 & 6.3 & 1 & & & & 1 & & & \\
\hline Sí & 132 & 132 & 6.0 & .96 & $.65-1.39$ & -.045 & .815 & .96 & $.66-1.41$ & -.037 & .851 \\
\hline \multicolumn{12}{|c|}{ Binge Drinking ${ }^{3}$} \\
\hline No & 1395 & 77 & 5.5 & 1 & & & & 1 & & & \\
\hline Sí & 1385 & 91 & 6.6 & 1.20 & $.88-1.65$ & .185 & .246 & 1.21 & $.88-1.65$ & .189 & .237 \\
\hline \multicolumn{12}{|l|}{ Audit } \\
\hline Negativo & 2279 & 115 & 5.1 & 1 & & & & 1 & & & \\
\hline Positivo & 501 & 53 & 10.6 & 2.23 & $1.58-3.13$ & .800 & .000 & 2.20 & $1.56-3.11$ & .789 & .000 \\
\hline
\end{tabular}

Nota. ${ }^{1}$ ORc $=$ Odds Ratio crudo $:{ }^{2} \mathrm{ORa}=$ Odds Ratio ajustado por Sexo, Edad y Titulación. ${ }^{3}$ Binge Drinking $=$ Consumo de cinco o más bebidas alcohólicas en el caso de los hombres y cuatro o más bebidas alcohólicas en el caso de las mujeres en una misma ocasión, de aproximadamente un par de horas. 
Tania Fernández-Villa, Juan Alguacil Ojeda, Ana Almaraz Gómez, José María Cancela Carral, Miguel Delgado-Rodríguez, Miguel GarcíaMartín, Eladio Jiménez-Mejías, Javier Llorca, Antonio José Molina, Rocío Ortíz Moncada, Luiz Félix Valero-Juan, Vicente Martín

Tabla 4

Tipos de utilización de Internet en usuarios problemáticos.

\begin{tabular}{|c|c|c|c|c|c|c|c|c|c|c|c|}
\hline & \multicolumn{11}{|c|}{ Uso Problemático de Internet } \\
\hline & $\mathbf{N}$ & $\mathbf{n}$ & $\%$ & $\mathrm{ORc}^{1}$ & IC95\% & B & $p$ & ORa ${ }^{2}$ & IC95\% & B & $p$ \\
\hline \multicolumn{12}{|l|}{ Chats } \\
\hline No & 1375 & 64 & 4.7 & 1 & & & & 1 & & & \\
\hline Sí & 1286 & 95 & 7.4 & 1.63 & $1.18-2.26$ & .491 & .003 & 1.60 & $1.14-2.23$ & .469 & .006 \\
\hline \multicolumn{12}{|l|}{ Email } \\
\hline No & 1908 & 126 & 6.6 & 1 & & & & 1 & & & \\
\hline Sí & 753 & 33 & 4.4 & .65 & $.44-.96$ & -.434 & .031 & .67 & $.45-.99$ & -.401 & .047 \\
\hline \multicolumn{12}{|l|}{ Juegos } \\
\hline No & 2454 & 141 & 5.8 & 1 & & & & 1 & & & \\
\hline Sí & 207 & 18 & 8.7 & 1.56 & $.94-2.61$ & .446 & .088 & 1.50 & $.89-2.54$ & .407 & .130 \\
\hline \multicolumn{12}{|c|}{ Redes Sociales } \\
\hline No & 885 & 37 & 4.2 & 1 & & & & 1 & & & \\
\hline Sí & 1776 & 122 & 6.9 & 1.69 & $1.16-2.46$ & .525 & .006 & 1.70 & $1.16-2.49$ & .532 & .006 \\
\hline \multicolumn{12}{|l|}{ Compras } \\
\hline No & 2578 & 151 & 5.9 & 1 & & & & 1 & & & \\
\hline Sí & 83 & 8 & 9.6 & 1.71 & $.81-3.62$ & .539 & .157 & 1.81 & $.85-3.83$ & .591 & .123 \\
\hline \multicolumn{12}{|l|}{ Prensa } \\
\hline No & 2250 & 142 & 6.3 & 1 & & & & 1 & & & \\
\hline Sí & 411 & 17 & 4.1 & .64 & $.38-1.07$ & -.445 & .090 & .64 & $.38-1.08$ & -.444 & .097 \\
\hline \multicolumn{12}{|l|}{ Series } \\
\hline No & 1517 & 87 & 5.7 & 1 & & & & 1 & & & \\
\hline Sí & 1144 & 72 & 6.3 & 1.10 & $.80-1.52$ & .099 & .547 & 1.08 & $.78-1.50$ & .080 & .628 \\
\hline
\end{tabular}

Nota. ${ }^{1}$ ORc $=$ Odds Ratio crudo $;{ }^{2} \mathrm{ORa}=$ Odds Ratio ajustado por Sexo, Edad y Titulación.

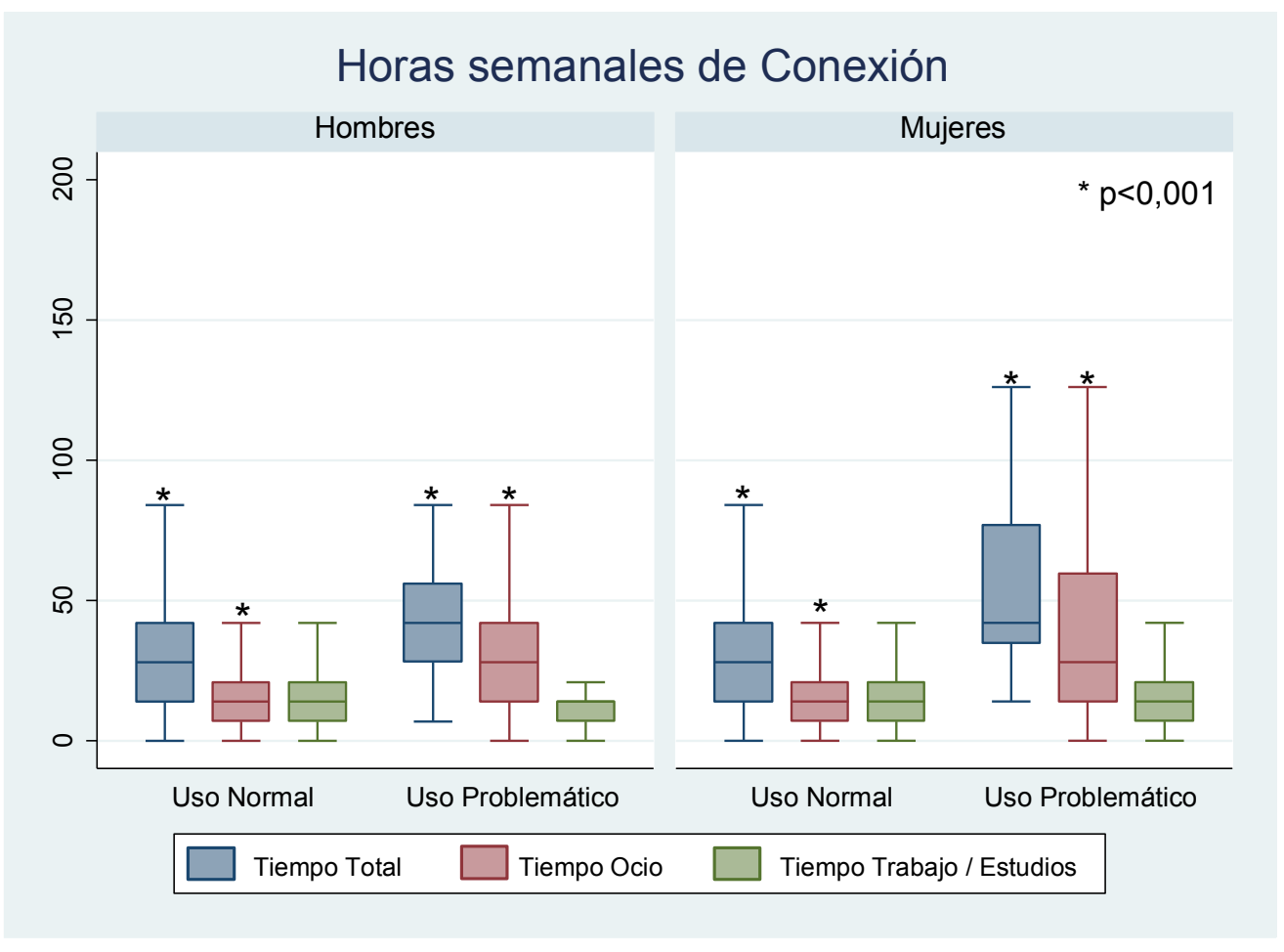

Figura 1. Horas semanales de conexión a Internet totales, por ocio y por trabajo o estudios.
Nota. En la representación gráfica la línea central de las cajas corresponde al percentil 50 o mediana, su altura viene establecida por el rango intercuartílico y las líneas externas explican la desviación de los datos 1.5 veces el rango intercuartílico. 
Tabla 5

Diferencias de género en función de los tipos de utilización de Internet en Usuarios Problemáticos.

\begin{tabular}{|c|c|c|c|c|c|c|c|c|c|c|c|c|}
\hline \multicolumn{13}{|c|}{ Hombres } \\
\hline & & $\mathbf{N}$ & $\mathrm{n}$ & $\%$ & $\mathbf{O R c ^ { 1 }}$ & IC95\% & B & $p$ & $\mathbf{O R a}{ }^{2}$ & IC95\% & B & $\mathbf{p}$ \\
\hline \multirow[t]{2}{*}{ Chats } & No & 463 & 33 & 7.1 & 1 & & & & 1 & & & \\
\hline & Sí & 327 & 16 & 4.9 & .67 & $.36-1.24$ & -.400 & .202 & .67 & $.36-1.25$ & -.401 & .208 \\
\hline \multirow[t]{2}{*}{ Email } & No & 574 & 39 & 6.8 & 1 & & & & 1 & & & \\
\hline & Sí & 216 & 10 & 4.6 & .67 & $.33-1.36$ & -.407 & .264 & .66 & $.32-1.35$ & -.414 & .258 \\
\hline \multirow[t]{2}{*}{ Juegos } & No & 670 & 36 & 5.4 & 1 & & & & 1 & & & \\
\hline & Sí & 120 & 13 & 10.8 & 2.14 & $1.10-4.17$ & .761 & .025 & 2.11 & $1.08-4.14$ & .747 & .029 \\
\hline \multirow[t]{2}{*}{ Redes Sociales } & No & 312 & 16 & 5.1 & 1 & & & & 1 & & & \\
\hline & Sí & 478 & 33 & 6.9 & 1.37 & $.74-2.54$ & .316 & .314 & 1.40 & $.75-2.59$ & .334 & .289 \\
\hline \multirow[t]{2}{*}{ Compras } & No & 775 & 46 & 5.9 & 1 & & & & 1 & & & \\
\hline & Sí & 15 & 3 & 20.0 & 3.96 & $1.08-14.53$ & 1.377 & .038 & 3.98 & $1.08-14.62$ & 1.381 & .038 \\
\hline \multirow[t]{2}{*}{ Prensa } & No & 583 & 40 & 6.9 & 1 & & & & 1 & & & \\
\hline & Sí & 207 & 9 & 4.4 & .62 & $.29-1.29$ & -.483 & .202 & .61 & $.29-1.29$ & -.492 & .195 \\
\hline \multirow[t]{2}{*}{ Series } & No & 438 & 25 & 5.7 & 1 & & & & 1 & & & \\
\hline & Sí & 352 & 24 & 6.8 & 1.21 & $.68-2.16$ & .190 & .521 & 1.20 & $.67-2.14$ & .184 & .532 \\
\hline \multicolumn{13}{|c|}{ Mujeres } \\
\hline & & $\mathbf{N}$ & $\mathbf{n}$ & $\%$ & ORc ${ }^{1}$ & IC95\% & B & $p$ & $\mathrm{ORa}^{2}$ & IC95\% & B & $p$ \\
\hline \multirow[t]{2}{*}{ Chats } & No & 912 & 31 & 3.4 & 1 & & & & 1 & & & \\
\hline & Sí & 959 & 79 & 8.2 & 2.55 & $1.67-3.90$ & .937 & .000 & 2.42 & $1.57-3.72$ & .882 & .000 \\
\hline \multirow[t]{2}{*}{ Email } & No & 1334 & 87 & 6.5 & 1 & & & & 1 & & & \\
\hline & Sí & 537 & 23 & 4.3 & .64 & $.40-1.03$ & -.444 & .065 & .67 & $.42-1.08$ & -.393 & .104 \\
\hline \multirow[t]{2}{*}{ Juegos } & No & 1784 & 10 & 5.9 & 1 & & & & 1 & & & \\
\hline & Sí & 87 & 5 & 5.8 & .98 & $.39-2.46$ & -.025 & .957 & .98 & $.39-2.48$ & -.018 & .970 \\
\hline \multirow[t]{2}{*}{ Redes Sociales } & No & 573 & 21 & 3.7 & 1 & & & & 1 & & & \\
\hline & Sí & 1298 & 89 & 6.9 & 1.93 & $1.19-3.14$ & .660 & .008 & 1.90 & $1.16-3.09$ & .640 & .010 \\
\hline \multirow[t]{2}{*}{ Compras } & No & 1803 & 105 & 5.8 & 1 & & & & 1 & & & \\
\hline & Sí & 68 & 5 & 7.4 & 1.28 & $.51-3.26$ & .250 & .600 & 1.36 & $.53-3.47$ & .307 & .520 \\
\hline \multirow[t]{2}{*}{ Prensa } & No & 1667 & 102 & 6.1 & 1 & & & & 1 & & & \\
\hline & Sí & 204 & 8 & 3.9 & .63 & $.30-1.31$ & -.468 & .212 & .66 & $.32-1.39$ & -.410 & .276 \\
\hline \multirow[t]{2}{*}{ Series } & No & 1079 & 62 & 5.8 & 1 & & & & 1 & & & \\
\hline & Sí & 792 & 48 & 6.1 & 1.06 & $.72-1.56$ & .057 & .775 & 1.03 & $.70-1.53$ & .033 & .866 \\
\hline
\end{tabular}

Nota. ${ }^{1} \mathrm{ORc}=$ Odds Ratio crudo $;{ }^{2} \mathrm{ORa}=$ Odds Ratio ajustado por Edad y Titulación.

\section{Discusión}

El uso de Internet se ha incrementado en los últimos años (AIMC, 2013; Internet World Stats, 2014), siendo varios los autores que relacionan esta herramienta con posibles estados de dependencia o adicción (Brenner, 1997; Echeburúa y Corral, 2010; Young, 1998). El escaso conocimiento de los problemas de salud que pueden derivarse de la sobreutilización de este medio, así como la disparidad de herramientas y criterios diagnósticos, justifican la necesidad de trabajos como este en el que no sólo se ha analizado la situación de la población universitaria en relación al uso de Internet dada su vulnerabilidad (Echeburúa y Corral, 2010;
Muñoz-Rivas et al., 2003); sino que se han identificado algunos de los posibles problemas de salud relacionados con su uso así como las diferencias de género existentes.

Nuestros resultados reflejan que seis de cada cien universitarios tienen problemas ocasionales o frecuentes con el uso de Internet, que pueden llegar a repercutir en su vida diaria o social, lo que está en consonancia con los datos establecidos por otros autores tanto para población adolescente como universitaria. En el estudio SEYLE llevado a cabo en once países europeos se encontró una prevalencia de uso problemático de Internet del $4.4 \%$ en adolescentes (Durkee et al., 2012). En población general Europea, las tasas ron- 
dan entre 1\% - 9\%. (Kaltiala-Heino, Lintonen y Rimpela, 2004; Siomos, Dafouli, Braimiotis, Mouzas y Angelopoulos, 2008;). Si nos referimos a población universitaria, las cifras se encuentran entre el $6 \%-40 \%$, siendo difícil establecer una comparativa dadas las diferencias geográficas y fundamentalmente metodológicas encontradas (Pezoa-Jares et al., 2012; Sussman et al., 2011).

Dentro de los factores de riesgo que se asocian a un UPI, destacan el sexo masculino, la edad inferior a 21 años, el domicilio fuera del hogar familiar, la baja autoestima, y la dependencia a otras conductas adictivas como es el consumo de drogas (Sánchez-Carbonell et al., 2008; Frangos et al., 2010; Muñoz-Rivas et al., 2003; Secades-Villa, 2014). A pesar de que nuestros resultados concuerdan en que son los menores de 21 años los que presentan mayores tasas de UPI, así como aquellos que cursan carreras de ramas diferentes a ciencias de la salud, no hemos encontrado diferencias estadísticamente significativas en cuanto al sexo.

En relación a posibles problemas de salud asociados con el UPI, Didia et al. (2009). establecieron relaciones con dolores de espalda, migrañas, perturbación del patrón del sueño, hábitos alimenticios irregulares y problemas familiares. Estos aspectos están en consonancia con los resultados del presente trabajo, donde existe una mayor prevalencia de UPI en personas con migrañas o dolores lumbares, no encontrándose asociación significativa con los dolores a nivel cervical. Nuestros datos también revelan una asociación positiva con el riesgo de trastornos de la conducta alimentaria y con el sobrepeso y obesidad.

Por otra parte, es importante resaltar el papel de la familia y la sociedad en el UPI, puesto que aquellos estudiantes que manifestaron haber sufrido algún tipo de discriminación o tenían un resultado positivo en el test APGAR presentaban mayores prevalencias.

Algunos autores describen además síntomas depresivos, tristeza o soledad como factores de riesgo de adicciones comportamentales, entre las que se enmarca la adicción a Internet (Alavi et al., 2012; Kaess et al., 2014). En este sentido, nuestros datos revelan que los estudiantes con riesgo de trastorno mental evaluado mediante el cuestionario GHQ12 presentan cuatro veces más UPI ( $\mathrm{ORa}=3.58$ IC95\%: 2.44 - 5.27), resaltando a su vez que aquellos que dicen sufrir algún trastorno depresivo duplican la frecuencia de este problema (ORa=2.22 IC95\%: $1.50-3.28)$.

Por otra parte, estudios previos han establecido una relación entre consumo de sustancias adictivas y el UPI (Pezoa-Jares et al., 2012; Secades-Villa, 2014; Sussman et al., 2011), lo que concuerda con nuestros datos para la relación de UPI con el uso problemático de alcohol valorado por el test AUDIT. Sin embargo, no se observó asociación entre UPI y el consumo de dichas sustancias (tabaco, alcohol, drogas ilegales...) algo que es explicable por el empleo de un criterio de clasificación que no discrimina si se trata de un uso puntual o de un uso problemático, lo que provoca la inclusión del grupo de usuarios problemáticos en un grupo mucho mayor de usuarios no problemáticos generando una regresión hacia el nulo de la asociación. Por lo tanto, nuestros resultados redundarían en la idea de que existe una asociación entre el UPI y otros comportamientos problemáticos, si bien no puede establecerse una direccionalidad en la relación existente dada la naturaleza transversal del estudio.

Respecto a la perturbación del patrón del sueño, es importante destacar no tanto las diferencias en cuanto a horas diarias de descanso (usuarios normales: 7.6 $\pm 1.2 \mathrm{~h} /$ día; Usuarios problemáticos: $7.3 \pm 1.5 \mathrm{~h}$ /día ; $\mathrm{p}=.003$ ), sino su calidad; puesto que aquellas personas que dicen tener un descanso inadecuado tienen dos veces más riesgo de sufrir este problema $(\mathrm{OR}=2.17 \mathrm{IC95 \%}: 1.58$-3.00), en relación a aquellas con un descanso suficiente.

En cuanto al tiempo de utilización, algunos estudios establecen diferencias entre usuarios problemáticos con una media semanal de 28 h de conexión y aquellos que no presentan problemas, cuya media de conexión semanal es de 12h (Yang y Tung, 2007). Nuestro estudio refleja tiempos relativamente superiores con una media de conexión semanal de $32 \mathrm{~h}$ en usuarios sin problemas y de $53 \mathrm{~h}$ en usuarios problemáticos, destacando especialmente el tiempo dedicado a actividades de ocio ( $19 \mathrm{~h}$ y $32 \mathrm{~h}$ respectivamente), siendo las mujeres las que más horas utilizan la Red a la semana.

En relación a otras posibles diferencias de género, hemos encontrado que los varones usan Internet principalmente para actividades relacionadas con el ocio como juegos online, o las compras, mientras que las mujeres se asocian más a actividades relacionadas con la socialización, como los chats y las redes sociales, lo que está en consonancia con lo publicado en la literatura científica (Andreassen et al., 2012; Muñoz-Rivas et al., 2003). Lo más destacable es que sean las mujeres las que más tiempo pasan conectadas a Internet, ya sea por motivos de ocio, trabajo o estudios, lo que difiere de lo encontrado en otros estudios, donde el sexo masculino se comporta como un factor de riesgo (Tsai et al, 2009; Leung y Lee, 2012).

El presente trabajo aporta información relevante en cuanto al uso de Internet en población universitaria, dado el escaso número de publicaciones al respecto. Sin embargo, los datos han de ser analizados con cautela, puesto que nuestro estudio no está exento de limitaciones. El carácter voluntario de la encuesta y la tendencia de los estudiantes a suavizar las conductas reprobables por la sociedad, conlleva un posible sesgo tanto de selección como de clasificación diferencial. Por otra parte, las diferencias metodológicas en cuanto al tipo de herramientas o criterios diagnósticos utilizados (IAT, GHQ-12, APGAR, SCOFF) pueden dificultar la comparabilidad con otros estudios y a su vez, el diseño descriptivo de este trabajo impide establecer relaciones causales.

Por ello, se plantean como futuras líneas de investigación, no solo analizar la situación momentánea de Internet con 
estudios descriptivos, sino también llevar a cabo seguimientos en el tiempo, como lo planteado en el diseño de cohorte del proyecto uniHcos, con el fin de determinar asociaciones causales con problemas de salud, consumo de drogas, cambios comportamentales relacionados con Internet e incluso modificaciones del rendimiento académico - profesional que pueden estar asociadas al tiempo de conexión.

\section{Reconocimientos}

Los autores de este trabajo quieren agradecer la colaboración de los estudiantes participantes y especialmente la financiación recibida a través de la Delegación del Gobierno para el Plan Nacional Sobre Drogas (Códigos: 2010|145 y 2013|034) para la realización de este estudio.

\section{Conflicto de Intereses}

Los autores de este artículo declaran no tener conflicto de intereses.

\section{Referencias}

AIMC. (2013). Asociación para la Investigación de Medios de Comunicación. 16 ${ }^{a}$ Encuesta: Navegantes en la Red. Recuperado de http://download.aimc.es/aimc/J5d8yq/ macro2013.pdf

Alavi, S., Ferdosi, M., Jannatifard, F., Eslami, M., Alaghemandan, H. y Setare, M. (2012). Behavioral Addiction versus Substance Addiction: Correspondence of Psychiatric and Psychological Views. International Journal of Preventive Medicine, 3, 290-294.

American Psichiatric Association. (2003). Diagnostic and statistical manual of mental disorders IV. Text revision. ( $4^{\mathrm{a}}$ ed.). Barcelona: Masson.

American Psychiatric Association. (2014). DSM-5 Development. Internet Gaming Disorder (Section III). Recuperado de http://www.dsm5.org/Documents/Internet\%20 Gaming\%20Disorder\%20Fact\%20Sheet.pdf

Andreassen, C., Torsheim, T., Brunborg, G. y Pallesen, S. (2012). Development of a Facebook addiction scale. Psychological Reports, 110, 1-17. Doi:10.2466/02.09.18. PR0.110.2.501-517

Bellón, J., Delgado, A., Del Castillo, J. y Lardelli, P. (1996). Validez y fiabilidad del cuestionario de función familiar Apgar-familiar. Atención Primaria, 18, 289-296.

Boletín Oficial del Estado. (1999). Ley Orgánica 15/1999 de Protección de Datos de Carácter Personal. BOE núm 298 de 14/12/1999. Recuperado de http://www.boe.es/boe/ dias/1999/12/14/pdfs/A43088-43099.pdf

Brenner, V. (1997). Psychology of computer use: Parameters of Internet use, abuse and addiction: The first 90 days of the Internet usage survey. Psicological Reports, 80, 879-882.
Carbonell, X. (2014). La adicción a los videojuegos en el DSM-5. Adicciones, 26, 91-95.

Didia, J., Dorphinghaus, A., Maggi, C. y Haro, G. (2009). Adicciones a Internet: Una posible inclusión en la nosografía. Revista de Psiquiatría del Uruguay, 73, 73-82.

Douglas, A., Mills, J., Niang, M., Stepchenkova, S., Byun, S., Ruffini, C.,... Blaton, M. (2008). Internet addiction: Meta-synthesis of qualitative research for the decade 1996 - 2006. Computers in Human Behavior, 24, 3027-3044. doi:10.1016/j.chb.2008.05.009

Durkee, T., Kaess, M., Carli, V., Parzer, P., Wasserman, C., Floderus, B.,... Wasserman, D. (2012). Prevalence of pathological Internet use among adolescents in Europe: demographic and social factors. Addiction, 107, 22102222. doi:10.1111/j.1360-0443.2012.03946.x.

Echeburúa, E. y Corral, P. (2010). Adicción a las nuevas tecnologías y a las redes sociales en jóvenes: un nuevo reto. Adicciones, 22, 91-95.

Fernández-Villa, T., Alguacil, J., Ayán, C., Bueno-Cavanillas, A., Cancela, J., Capelo, R.,... Martín, V. (2013). Proyecto UNIHCOS. Cohorte dinámica de estudiantes universitarios para el estudio del consumo de drogas y otras adicciones. Revista Española de Salud Pública, 87, 575-585. doi:10.4321/S1135-57272013000600003.

Frangos, C., Frangos, C. y Kiohos, A. (2010). Internet Addiction among Greek University Students: Demographic Associations with the Phenomenon, Using the Greek Version of Young's Internet Addiction Test. International Journal of Economic Sciences and Applied Research, 3, 49-74.

Garcia-Campayo, J., Sanz-Carrillo, C, Ibañez, J. A., Lou, S., Solano, V. y Alda, M. (2005). Validation of the Spanish version of the SCOFF questionnaire for the screening of eating disorders in primary care. Journal of Psychosomatic Resarch, 59, 51-55.

Goldberg, D. P., Gater, R., Sartorius, N., Ustun, T. B., Piccinelli, M., Gureje, O. y Rutter, C. (1997). The validity of two versions of the GHQ in the WHO study of mental health illness in general health care. Psychological Medicine, 27, 191-197

Glowniag, J. (1998). History, structure, and function of the Internet. Seminars in Nuclear Medicine, 28, 135-44.

Gracia, M., Vigo, M., Fernández, M. J. y Marco, M. (2002). Problemas conductuales relacionados con el uso de Internet: un estudio exploratorio. Anales de Psicología, 18, 273-292.

Hicks, T. y Heastie, S. (2008). High school to college transition: a profile of the stressors, physical and psychological health issues that affect the first-year on campus college student. Journal of Cultural Diversity, 15, 143-147.

Internet World Stats. (2014). Usage and population statistics. Recuperado de http://www.Internetworldstats. com/

Jelenchick, L., Becker, T. y Moreno, M. (2012). Assessing the psychometric properties of the Internet Addiction 
Test (IAT) in US college students. Psychiatry Research, 196, 296-301. doi:10.1016/j.psychres.2011.09.007.

Kaess, M., Durkee, T., Brunner, R., Carli, V., Parzer, P., Wasserman, C.,... Wasserman, D. (2014). Pathological Internet use among European adolescents: psychopathology and self-destructive behaviours. European Child E Adolescent Psychiatry, 23, 1093-1102. doi: 10.1007/ s00787-014-0562-7

Kaltiala-Heino, R., Lintonen, T. y Rimpela, A. (2004). Internet addiction? Potentially problematic use of the Internet in a population of 12-18 year-old adolescents. Addiction Research $\mathcal{E}$ Theory, 12, 89-96. doi:10.1080/1606635031000098796

Kandell, J. J. (1998). Internet addiction on campus: The vulnerability of college students. CyberPsychology $\mathcal{E} \mathrm{B} e$ havior, 1, 11-17. doi:10.1089/cpb.1998.1.11.

Lam-Figueroa, N., Contreras-Pulache, H., Mori-Quispe, E., Nizama-Valladolid, M., Gutiérrez, C., Hinostroza-Camposano, W.,... Hinostroza-Camposano, W. D. (2011). Adicción a Internet: Desarrollo y Validación de un instrumento en escolares adolescentes de lima, Perú. Revista Peruana de Medicina Experimental y Salud Pública, 28, 462-69.

Ledo-Varela, M., Luis, D., González-Sagrado, M., Izaola, O., Conde, R. y Aller, R. (2011). Características nutricionales y estilo de vida en universitarios. Nutrición Hospitalaria, 26, 814-818.

Leung, L. y Lee, P. (2012). The influences of information literacy, Internet addcition and parenting styles on Internet risks. New Media E Society, 14, 117-136. doi:10.1177/1461444811410406

Luengo, A. (2004). Adicción a Internet: conceptualización y propuesta de intervención. Revista Profesional Española de Terapia Cognitivo-Conductual, 2, 22-52.

Morgan, J., Reid, F. y Lacey , J. (1999). The SCOFF questionnaire: assessment of a new screening tool for eating disorders. British Medical Journal, 319, 1467-1468.

Muñoz-Rivas, M., Navarro, M. E. y Ortega, N. (2003). Patrones de uso de Internet en población universitaria. Adicciones, 15, 137-144.

Organización Mundial de la Salud. (1992). CIE-10. Trastornos mentales y del Comportamiento. Madrid: MEDITOR.

Pezoa-Jares, R., Espinoza-Luna, I. L. y Vasquez-Medina, J. A. (2012). Internet Addiction: A Review. Journal of Addiction Research $\mathcal{E}$ Therapy, S6, 004. doi:10.4172/2155-6105. S6-004

Rocha, K., Pérez, K., Rodríguez, M., Borrell, C. y Obiols, J. (2011). Propiedades psicométricas y valores normativos del General Health Questionnaire (GQH-12) en la población general española. International Journal of Clinical and Health Psychology, 11, 125-139.

Sánchez-Carbonell, X., Beranuy, M., Castellana, M., Chamarro, A. y Oberst, U. (2008). La adicción a Internet y al móvil: ¿̇moda o trastorno?. Adicciones, 20, 149160 .
Saunders, J. B., Aasland, O. G., Babor, T. F., de la Fuente, J. R. y Grant, M. (1993). Development of the Alcohol Use Disorders Identification Test (AUDIT): WHO collaborative project on early detection of persons with harmful alcohol consumption. II. Addiction, 88, 791-804.

Spada, M. (2014). An overview of problematic Internet use. Addictive Behaviors, 39, 3-6.

Secades-Villa, R., Calafat, A., Fernández-Hermida, J. R., Juan, M., Duch, M., Skärstrand, E.,... Talic, S. (2014). Duration of Internet use and adverse psychosocial effects among European adolescents. Adicciones, 26, 247253.

Siomos, K. E., Dafouli, E. D., Braimiotis, D. A., Mouzas, O. D. y Angelopoulos, N. V. (2008). Internet addiction among Greek adolescent students. CyberPsychology $\mathcal{E}$ Behavior, 11, 653-657. doi:10.1089/cpb.2008.0088.

Smilkstein, G., Ashworth, C. y Montano D. (1982). Validity and reliability of the Family APGAR as a test of family function. Journal of Family Practice, 15, 303-311

Stata Corp. (2013). Stata Statistical Software: Release 13. College Station, TX: StataCorp LP.

Sussman, S., Lisha, N. y Griffiths, M. (2011). Prevalence of the Addictions: A Problem of the Majority or the Minority? Evaluation $\mathcal{E}$ the Health Professions, 34, 3-56. doi:10.1177/0163278710380124.

Tsai, H. F., Cheng, S. H., Yeh, T. L., Shih, C. C., Chen, K. C., Yang, Y. C. y Yang , Y. K. (2009). The risk factors of Internet addiction - A survey of university freshmen. Psychiatry Research, 167, 294-299. doi:10.1016/j.psychres.2008.01.015

Yang, S. C. y Tung, C. (2007). Comparison of Internet addicts and non-addicts in Taiwanese high school. Computers in Human Behavior, 23, 79-96. doi:10.1016/j. chb.2004.03.037

Young, K. S. (1998). Internet Addiction: The emergence of a new clinical disorder. CyberPsychology $\mathcal{E}$ Behavior, 1, 237-244.

Young, K. (2010). Internet addiction over the decade: a personal look back. World Psychiatry, 9, 91. 